\title{
Immunological hotspots analyzed by docking simulations: evidence for a general mechanism in pemphigus vulgaris pathology and transformation Joo Chuan Tong*1 and Animesh A Sinha ${ }^{2}$
}

Address: ${ }^{1}$ Data Mining Department, Institute for Infocomm Research, 21 Heng Mui Keng Terrace, 119613, Singapore and ${ }^{2}$ Center for Investigative Dermatology, Division of Dermatology and Cutaneous Sciences, College of Human Medicine, Michigan State University, 4120 Biomedical and Physical Sciences Building, East Lansing, MI 48824, USA

Email: Joo Chuan Tong* - jctong@i2r.a-star.edu.sg; Animesh A Sinha - asinha@msu.edu

* Corresponding author

Published: 19 June 2008

BMC Immunology 2008, 9:30 doi:10.1 186/147/-2172-9-30
Received: 15 January 2008

Accepted: 19 June 2008

This article is available from: http://www.biomedcentral.com/I47I-2/72/9/30

(c) 2008 Tong and Sinha; licensee BioMed Central Ltd.

This is an Open Access article distributed under the terms of the Creative Commons Attribution License (http://creativecommons.org/licenses/by/2.0), which permits unrestricted use, distribution, and reproduction in any medium, provided the original work is properly cited.

\begin{abstract}
Background: Pemphigus vulgaris (PV) is an acquired autoimmune blistering disorder in which greater than $80 \%$ of active patients produce autoantibodies to the desmosomal protein desmogelin 3 (Dsg3). As the disease progresses, $40-50 \%$ of patients may also develop reactivity to a second component of the desmosomal complex, desmogelin I (Dsgl). T cells are clearly required for the production of autoantibodies in PV. However, few T-cell specificities within Dsg3 or Dsgl have been reported to date, and the precise role of T-cells in disease pathogenesis and evolution remains poorly understood. In particular, no studies have addressed the immunological mechanisms that underlie the observed clinical heterogeneity in pemphigus. We report here a structure-based technique for the screening of DRBI*0402-specific immunological (T-cell epitope) hotspots in both Dsg3 and Dsgl glycoproteins.

Results: High predictivity was obtained for DRBI*0402 $\left(r^{2}=0.90, s=1.20 \mathrm{~kJ} / \mathrm{mol}, q^{2}=0.82\right.$, $s_{\text {press }}$ $=1.61 \mathrm{~kJ} / \mathrm{mol}$ ) predictive model, compared to experimental data. In silico mapping of the T-cell epitope repertoires in Dsg3 and Dsgl glycoproteins revealed that the potential immunological hotspots of both target autoantigens are highly conserved, despite limited sequence identity (54\% identical, $72 \%$ similar). A similar number of well-conserved (18\%) high-affinity binders were predicted to exist within both Dsg3 and Dsgl, with analogous distribution of binding registers.

Conclusion: This study provides interesting new insights into the possible mechanism for PV disease progression. Our data suggests that the potential T-cell epitope repertoires encoded in Dsgl and Dsg3 is substantially overlapping, and it may be possible to apply a common, antigenspecific therapeutic strategy with efficacy across distinct clinical phases of disease.
\end{abstract}

\section{Background}

Pemphigus vulgaris (PV) is characterized by the loss of normal epithelial cell-to-cell adhesion leading to blistering which may involve the mucous membranes, non- mucosal cutaneous surfaces, or both [1]. Pemphigus autoantibodies (autoAb) are mainly directed against the desmosomal glycoproteins desmoglein 3 (Dsg3) and des- 
moglein 1 (Dsg1), members of the cadherin superfamily of cell adhesion molecules [2].

Clinical evolution of disease expression is common in PV $[3,4]$. In early disease, a majority of PV patients develop autoantibodies to Dsg3 coincident with mucosal blisters. In later stages, significant proportions of patients develop additional lesions on non-mucosal cutaneous sites and exhibit non-cross-reactive immunity to both Dsg3 and Dsg1 [5].

Two immunologic phenomenon termed "antigen mimicry" [5] and "epitope spreading" [5-8] have been proposed as possible pathogenic mechanisms responsible for the shift in autoreactive lymphocyte (T- or B-cell) profile from Dsg3+/Dsg1- to Dsg3+/Dsg1+. Antigen mimicry can be defined as the generation of lymphocyte (T- or B-cell) reactivity towards a protein due to its close structural similarity to unique exogenous antigens, or new determinants that have been generated endogenously [5]. Epitope spreading in the context of autoimmunity refers to the development of epitope-specific immune responses that are distinct from and non-cross-reactive with diseaseinducing epitopes on the same (or different) protein secondary to the release of such a self-protein during an autoimmune response [8-10].

A close relationship between antigen mimicry and epitope spreading exists, with epitope spreading usually occurring after an initial episode of antigen mimicry [5]. Exogenous and endogenous antigens that may trigger cross-reactivity with self-proteins have not yet been defined in pemphigus [5]. While the modulation of autoantibody reactivities in the transformation of one disease subform into another has been actively explored [3-7], the role of T-cells underlying the evolution of autoreactive processes and epitope spreading remains poorly understood. To date, limited studies on T-cell specificities within PV have been reported [11-20]. The reported HLA associations with disease may serve to provide the genetic link that drives the evolving autoimmune responses in pemphigus. $\mathrm{PV}$ is known to be strongly associated with the HLA-DR allele DRB1*0402 [21-26]; it is present in more than $90 \%$ of Ashkenazi patients [27]. The DRB $1{ }^{*} 0402$ allele is also common in other ethic backgrounds, including patients from France [28], Italy [29], Spain [30], Argentina [31] and Iran [32].

We have previously investigated the docking potentials of Dsg3 peptides to DRB1*0402 using a hybrid approach that integrates the strength of Monte Carlo simulations and homology modeling [33-37]. Consistent with experimental evidence [11], computational simulations reveal that a potentially large number of T-cell epitopes may be relevant in the pathogenesis of PV [33]. In the current study, we have extended our analysis to the Dsg1 glycoprotein and applied a new scoring scheme for identification of immunological (T-cell epitope) hotspots within both Dsg3 and Dsg1 self-antigens. In silico mapping of the T-cell epitope repertoires within Dsg3 and Dsg1 suggests that similar peptides from both PV target antigens may be involved in disease progression and the evolution in autoreactive lymphocyte reactivity during the course of disease from one clinical subtype to another (mucosal PV to mucocutaneous PV).

\section{Results and Discussion \\ Comparison of Dsgl and Dsg3 Extracellular Domains}

The Dsg3 extracellular domain (ECD) has an extensive surface area of $32133 \AA 2$. This surface area is 3\% larger than the Dsg1 ECD atomic accessible surface (31093 $\AA^{2}$ ). The general folds of Dsg1 and Dsg3 ECDs are similar (Figures 1 and 2). In particular, the ECD1, ECD2 and ECD3 of Dsg1 and Dsg3 are well conserved with C $\alpha$ r.m.s.d. of 1.03 $\AA$, $1.09 \AA$ and $1.94 \AA$, respectively. The main differences between Dsg1 and Dsg3 ECDs lie in ECD4 (C $\alpha$ r.m.s.d. = $3.76 \AA$ ) and ECD5 (C $\alpha$ r.m.s.d. = $6.95 \AA$ ). In ECD4, the most obvious difference between the structures involve the loop 411-420 of Dsg1 and loop 405-422 of Dsg3. The backbone of Gln410, Ala411 and Ile412 in Dsg1 are intercalated more deeply into the ECD4 interface than the corresponding residues in Dsg3. In ECD5, the loop 468479 curled upward toward Dsg1, with differences of up to $2.18 \AA$ between the corresponding positions in Dsg3 (loop 468-475). It appears that these regions may not contain high concentration of T-cell epitopes and do not contribute directly to the differences in T-cell specificities of the two PV target antigens (Figure 3). Nonetheless, these structural differences are solvent exposed and may interact with pemphigus autoAb.

\section{Dsgl and Dsg3 Share Common Immunological Hotspots}

The locations of Dsg1 and Dsg3 immunological hotspots are highly conserved across the ECD, TM and intracellular domain (ICD), despite limited sequence identity (54\% identical, $72 \%$ similar). There are substantial overlaps between predicted and known immunological (T-cell epitope) hotspots (Dsg3 145-192, Dsg3 240-303, Dsg3 $570-614$ ) at the threshold $-123 \mathrm{~kJ} / \mathrm{mol}$ (Figure 2). At this threshold, the number of predicted Dsg3 hotspots are ten (residues 21-88, 125-147, 173-221, 245-299, 330-391, $435-456,495-522,584-640$, 772-797, 830-859). Among these, one spans the signal peptide (SP), propeptide (PP) and the ECD; six are present in the ECD; one extends across the ECD, TM and ICD; while two hotspots are predicted to exist in the ICD (Figure 2). Similarly, nine hotspots were predicted for Dsg1 (residues 1-62, 82-151, 173-240, 310-372, 423-455, 470-498, 550-569, 656$688,732-760)$, all of which overlap with those from Dsg3 (Figure 2). Collectively, these results suggest that the 


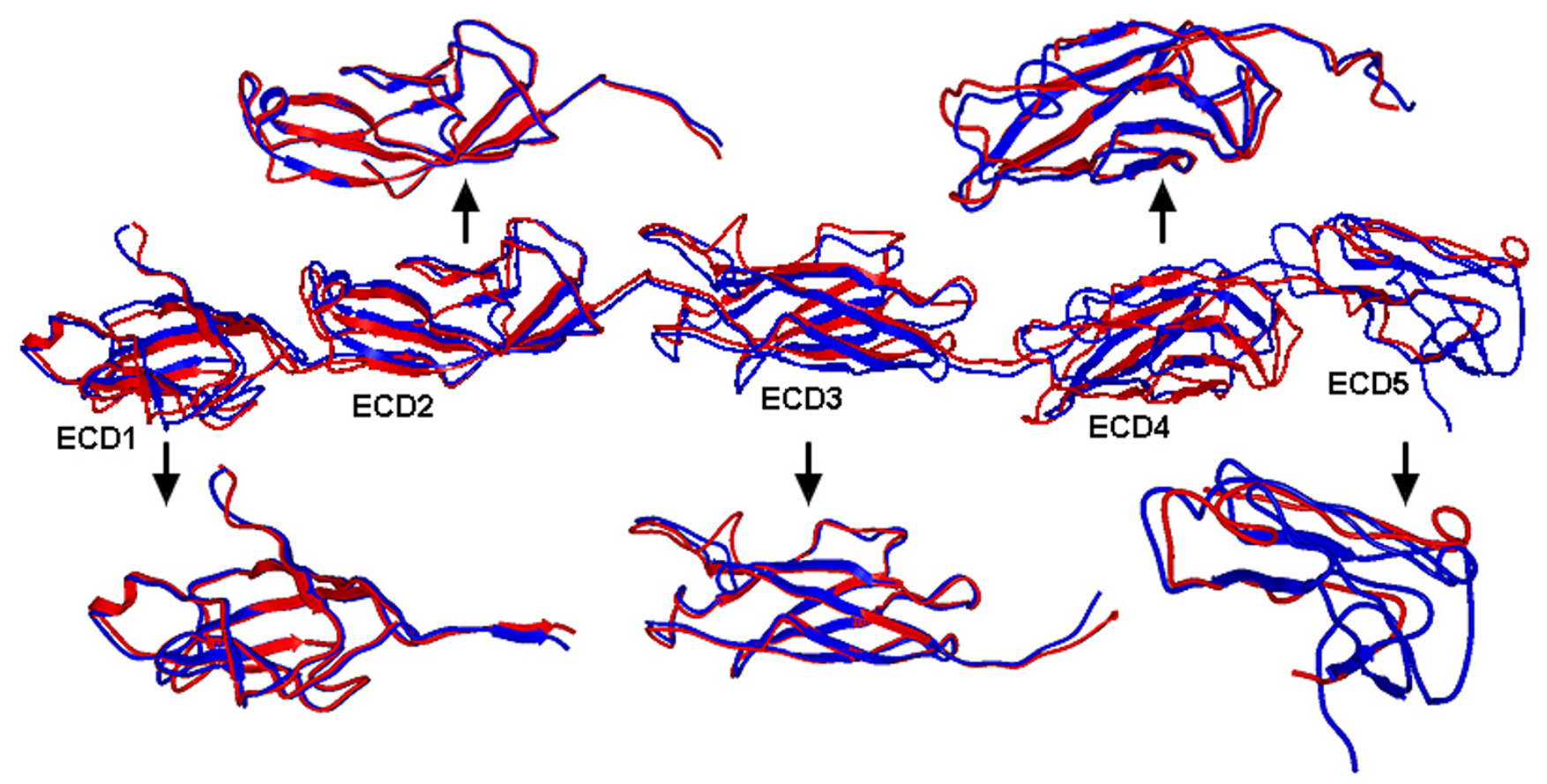

Figure I

Superposition of the structures of Dsg I and Dsg3 extracellular domains. Structural comparison of the Dsg I (red) and Dsg3 (blue) extracellular domains. The r.m.s.d. of the entire ECD, ECDI, ECD2, ECD3, ECD4, and ECD5 are $5.56 \AA$, I.03 $\AA$, I.09 $\AA$, I.94 $\AA$, $3.76 \AA$, and $6.95 \AA$, respectively.

potential T-cell epitope repertoire encoded in Dsg1 and Dsg3 is substantially overlapping, and may help to explain the molecular basis underlying the observed intermolecular spreading from Dsg3 to Dsg1 targets during the course of PV.

\section{Effects of Binding Registers in Peptide Selection}

We investigated the effect of multiple contact regions or binding registers in Dsg3 peptides specific to DRB1*0402 [33]. Among 985 Dsg3 peptide sequences (including SP and PP derived sequences), 658 were predicted high-affinity binders with $77 \%$ containing two or more registers that can be docked into the binding groove of DRB1*0402. A similar number of high-affinity binders are predicted to exist within the Dsg1 glycoprotein, with analogous distribution of binding registers as illustrated in Figure 4. Of 1035 Dsg1 peptides, 665 were predicted high-affinity binders with 129 ( 23\%), 112 ( 19\%), 130 ( 17\%), 99 ( $\sim 15 \%)$ and 43 ( $\sim 6 \%)$ peptides possessing two, three, four, five and six registers, respectively.

\section{Redundancy Profiles of Predicted Binding Peptides}

The predicted Dsg1 high-affinity binding sequences were examined for their similarity with the Dsg3 proteome. Each Dsg1 (15 mer) sequence was used to probe the entire Dsg3 proteome for the highest identity Dsg3 (15 mer) peptide with the minimal number of substitutions. Figure 5 details the degree of conservation of Dsg1 predicted high-affinity binders with Dsg3 sequences. All predicted Dsg1 and Dsg3 (15 mer) binding peptides share at least four common residues along their primary sequences. It has been reported that PV and PF autoAbs can cross-react with Dsg1 and Dsg3 peptides with 75\% identity [38]. Sequence alignment showed that $18 \%$ (or 122) of these peptides are highly conserved with at least $75 \%$ sequence identity. In this context, two peptides Dsg1 $1_{58-72}$ CREGEDNSKRNPIAK and Dsg1 $1_{59-73}$ REGEDNSKRNPIAKI near the N-terminus of ECD1 appear to be of particular interest, since they are fully conserved within the Dsg3 proteome and may represent the most likely antigenic link between self-directed responses to two distinct autoantigens (Dsg3 and Dsg1). A subset of patients with PV has been shown to have T cell reactivity to both Dsg3 and Dsg1 [39]. However, defined Dsg1 epitopes have not yet been determined.

\section{Conclusion}

Although clinical evolution of disease expression is common in PV [3,4], our understanding of how T-cells are involved in the underlying autoreactive processes remains poor. Collectively, the results of this study provide interesting new insights into the possible mechanism of dis- 


\section{Dsg1}
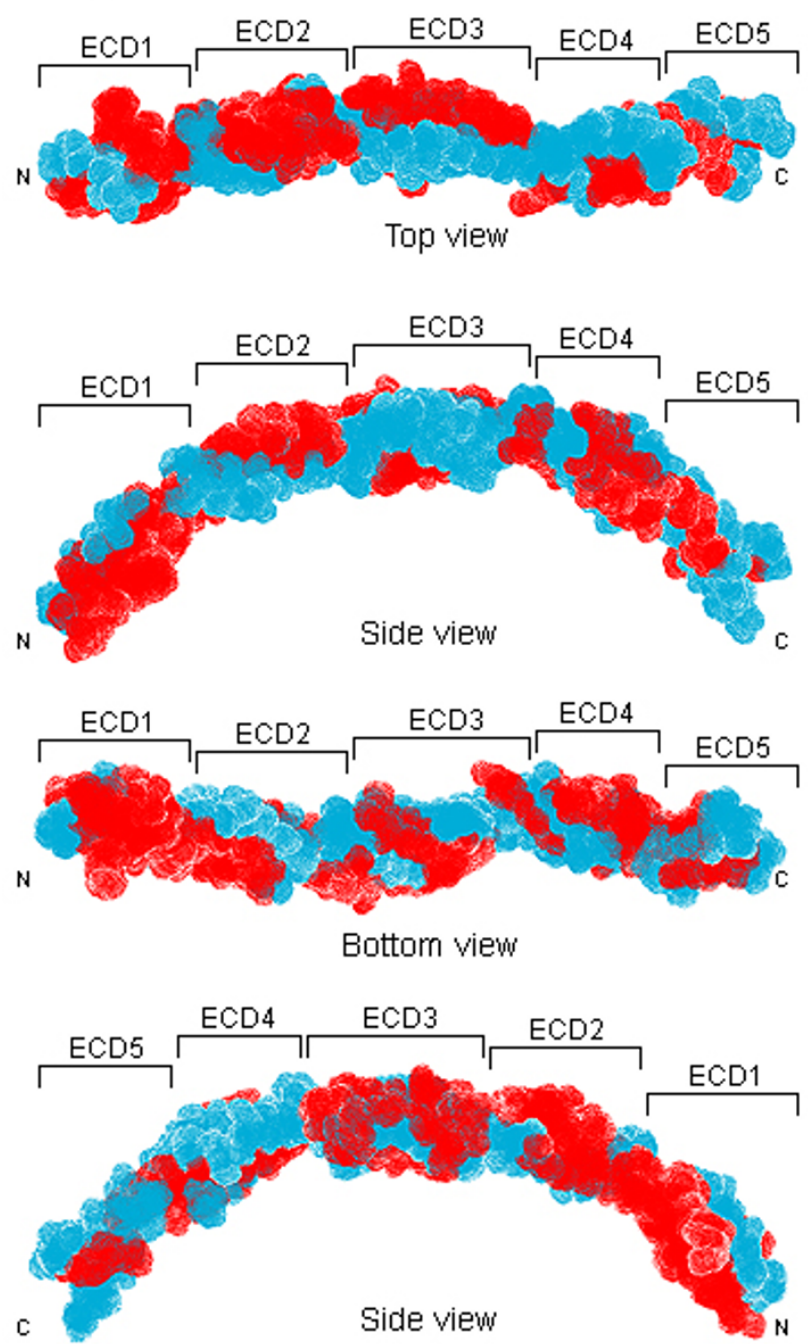

\section{Dsg3}
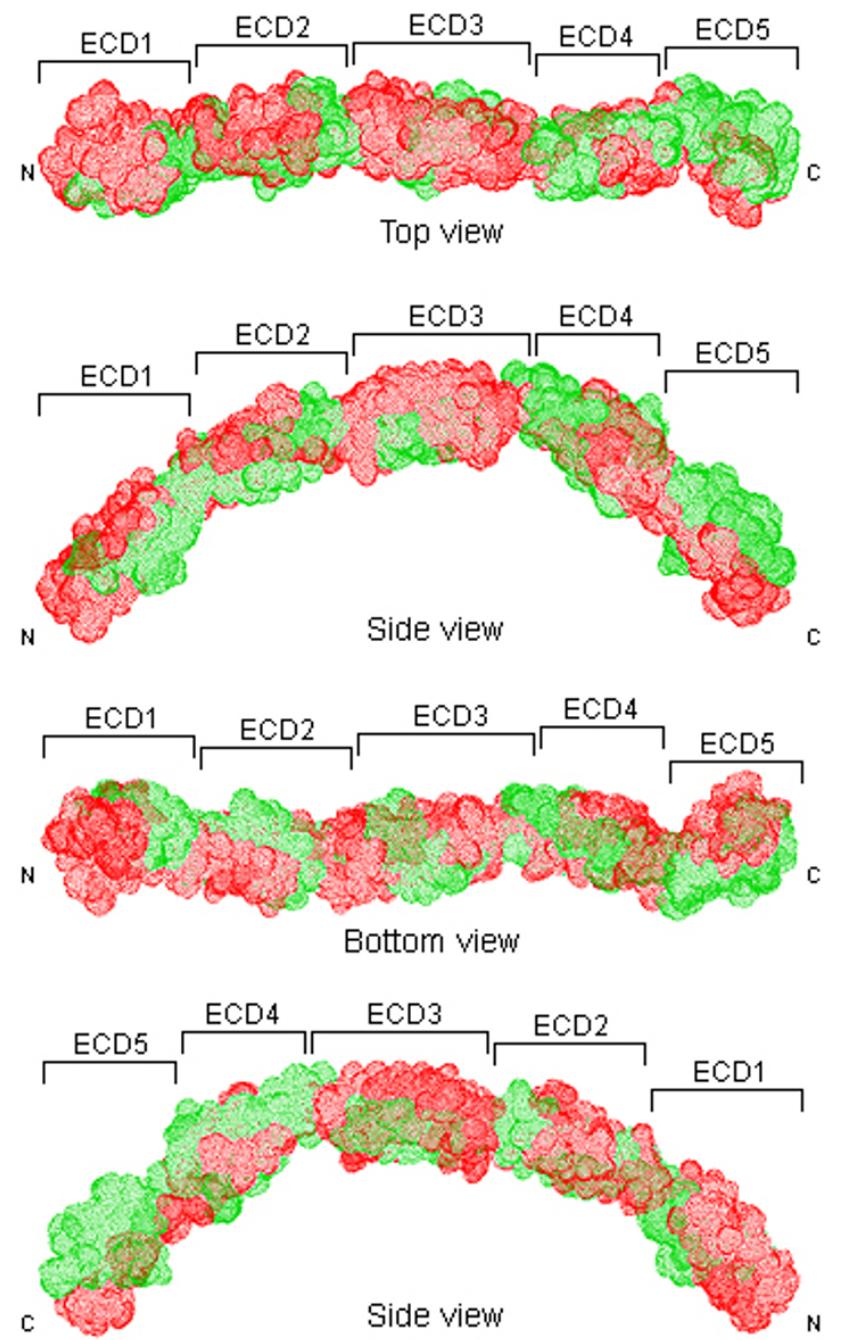

Figure 2

Molecular surface of Dsg I and Dsg3 extracellular domains illustrating the positions of predicted DRB I*0402specific immunological (T-cell epitope) hot spots. Locations of predicted immunological hot spots (colored red) for Dsgl and Dsg3 extracellular domains are shown in surrounding views and numbered in accordance to their extracellular domains.

ease progression. Our data suggests that similar peptides from the two known PV autoantigenic targets may bind to DRB $1 * 0402$ and allow for intermolecular epitope spreading that lead to distinct morphological categories of PV mucosal lesions only $v s$. mucocutaneous disease. Nonetheless, many other factors exist, such as IgG-activated intracellular signalling events [40], which may play a critical role in the complex disease mechanism and should also be explored. Recently, Lucchese and coworkers [38] discovered an immunodominant Dsg3 T-cell epitope Dsg $3_{49-60}$ REWVKFAKPCRE that is highly reactive with both PV and PF sera, with $75 \%$ identity to Dsg $1_{49-60}$
REWIKFAAACRE. In this context, our current approach of epitope mapping may prove useful in facilitating the systematic discovery of peptides that cross-react with both PV and PF autoreactive T-cells and serve as targets for potential therapeutic approaches that are efficient in both diseases. Further studies are necessary to determine the proportion of immunogenic epitopes that are capable of stimulating autoreactive lymphocytes from both pemphigus subtypes. 

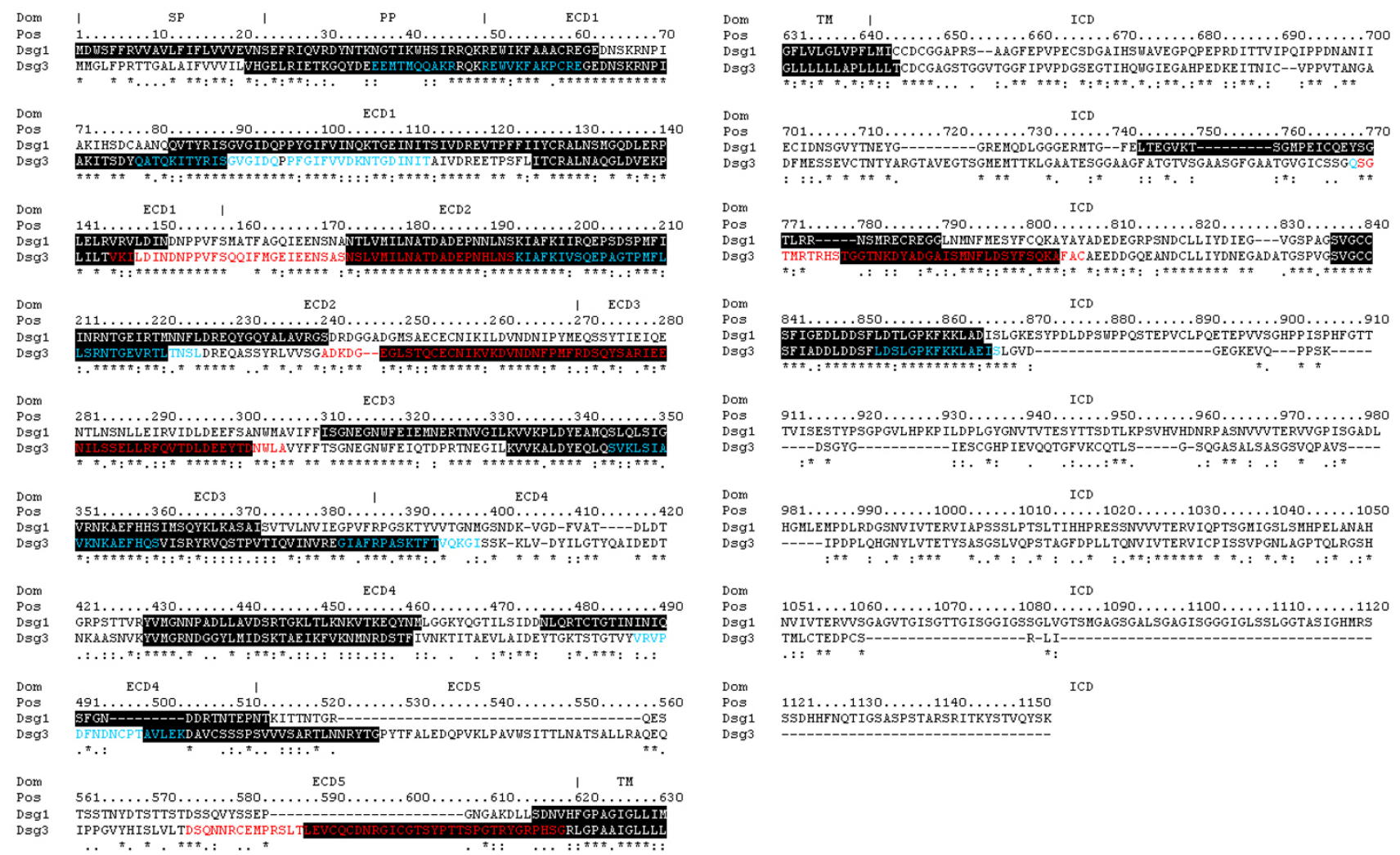

Figure 3

Predicted DRB I*0402-specific immunological hot spots for Dsg I and Dsg3 glycoproteins. Multiple sequence alignment of Dsgl and Dsg3. Predicted hot spots (threshold $=-123 \mathrm{~kJ} / \mathrm{mol}$ ) shaded in black, experimental Dsg3 hot spots highlighted red, and experimentally confirmed DRBI*0402-specific Dsg3 peptides highlighted blue. Dsg I - Signal Peptide, SP: I-23; Propeptide, PP: 24-49; Extracellular domain I, ECDI: 50-I58; ECD2: I59-270; ECD3: 27I-385; ECD4: 386-497; ECD5: 498548; Transmembrane, TM: 549-569; Intracellular domain, ICD: 570-I049. Dsg3 - SP: I-23; PP: 24-49; ECDI: 50-I58; ECD2: |59-268; ECD3: 269-383; ECD4: 386-499; ECD5: 500-6I5; TM: 616-640; ICD: 64|-999.

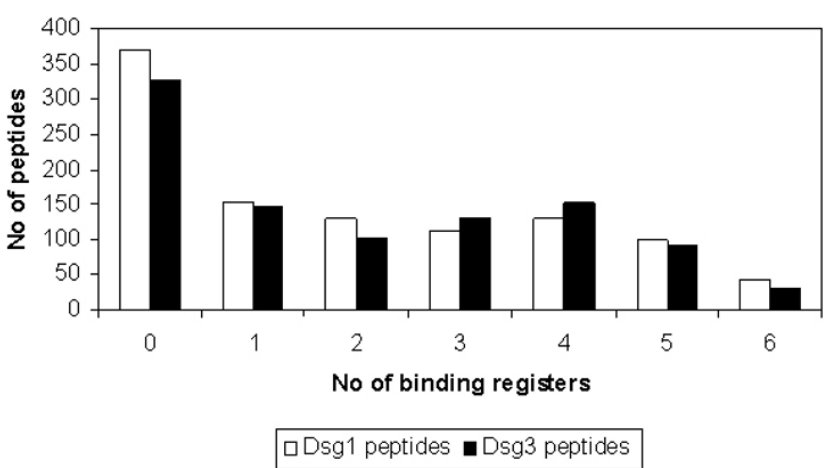

Figure 4

Number of predicted binding registers for Dsg I and Dsg3 peptides bound to DRB I*0402. The frequency of DsgI (colored white) and Dsg3 (colored black) peptides docked to DRBI*0402 shown as a function of the number of predicted binding registers.

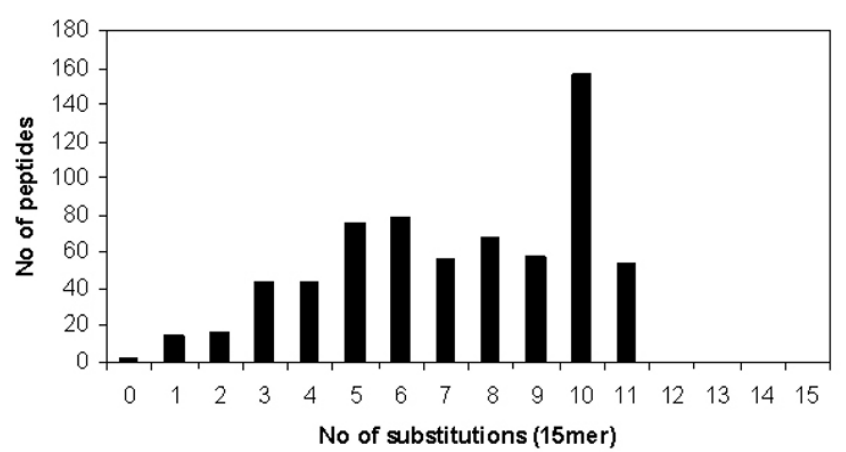

Figure 5

Redundancy profile of predicted Dsg I peptides to Dsg3 sequences. The number of predicted high-affinity Dsg I (I 5 mer) peptide sequences shown as a function of the number of substitutions from the highest identity peptide sequence derived from the Dsg3 proteome derived using a 15 mer sliding window. 


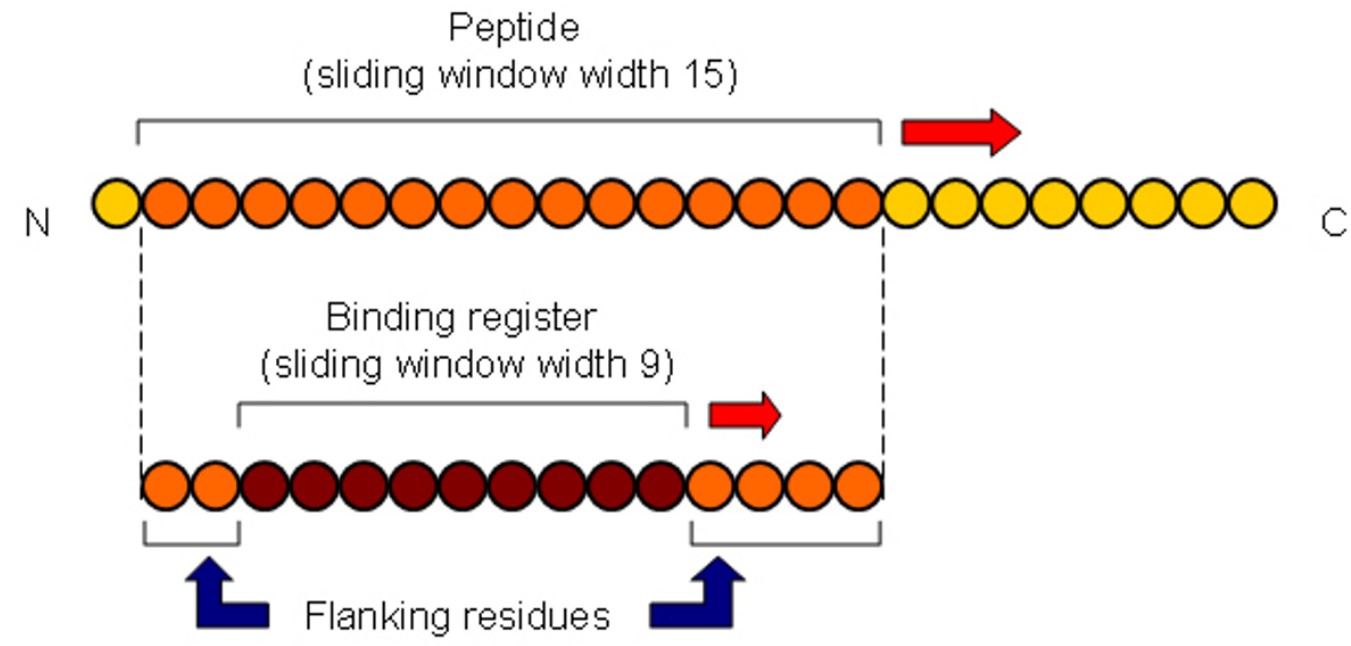

Figure 6

Generation of Dsg I and Dsg3 peptide sequences for docking into DRB I*0402. Overlapping I5 mer peptides are generated from the Dsgl and Dsg3 sequences. For each 15 mer peptide, an overlapping sliding window of size nine is applied to generate all combinations of binding registers to be modeled into the binding groove of DRBI*0402.

\section{Methods}

The sequence of DRB1*0402 was obtained from IMGTHLA database [41]. Dsg1 and Dsg3 sequence data were obtained from Swiss-Prot [42]. To identify suitable structural templates in the Protein Data Bank (PDB) [43] for model construction, a sequence similarity search was performed using BLASTP [44] and the highest quality templates (with the best resolution, highest sequence similarity and minimal number of missing residues) were selected among the returned hits. The crystal structure of DRB1*0401 (PDB code 1D5Z) was selected as template for DRB1*0402 (97.9\% identity) [33], while the solution structures of C-cadherin (PDB code 1Q5C) and N-cadherin (PDB code $1 \mathrm{NCI}$ ) were used for constructing the extracellular domains of both Dsg1 and Dsg3.

\section{Model Building}

The program MODELLER [45] was employed for comparative modeling of DRB1*0402, Dsg1 and Dsg3. Each model was constructed by optimally satisfying spatial constraints obtained from the alignment of the template structure with the target sequence and from the CHARMM-22 force field [46], and subsequently relaxed by conjugate gradient minimization, using the program Internal Coordinate Mechanics (ICM) $[47,48]$.

\section{Peptide Docking}

Overlapping 15 mer peptides are generated from the Dsg1 and Dsg3 sequences. An overlapping sliding window of size nine is applied to each 15 mer peptide to generate all combinations of binding registers to be modeled into the binding groove of DRB1*0402 (Figure 6). A total of 1035
Dsg1 15 mer peptides (6210 binding registers) and 985 Dsg3 15 mer peptides (5910 binding registers) were generated and used in the current analysis. Docking was performed using a standard protocol as previously described [33-35], consisting of (i) pseudo-Brownian rigid body docking of peptide fragments to the ends of the binding groove, (ii) central loop closure by satisfaction of spatial constraints, (iii) refinement of the backbone and sidechain atoms of the core recognition residues and receptor contact regions within $4.00 \AA$ A radius, and (iv) extension of flanking peptide residues by satisfaction of spatial constraints (Figure 7).

\section{Empirical Free Energy Functions}

The scoring function presented in this study is based on the free energy potential in ICM $[47,48]$. Computation of the binding free energy was performed according to previous similar works $[36,37]$ based on the difference between the energy of the solvated complex and the sum of the energy of the solvated receptor and that of the peptide ligand, followed by optimization using experimental $\mathrm{IC}_{50}$ values. The DRB1*0402 model optimization, training and testing were described earlier [33].

\section{Immunological Hotspot Prediction}

In the present study, 'immunological hotspots' are defined as antigenic regions of up to 30 amino acids based on the sum of predicted binding energies of the top four binders within a window of 30 amino acids $[49,50]$. Where available, predicted hotspots were validated using available experimentally determined sites (Figure 3). 


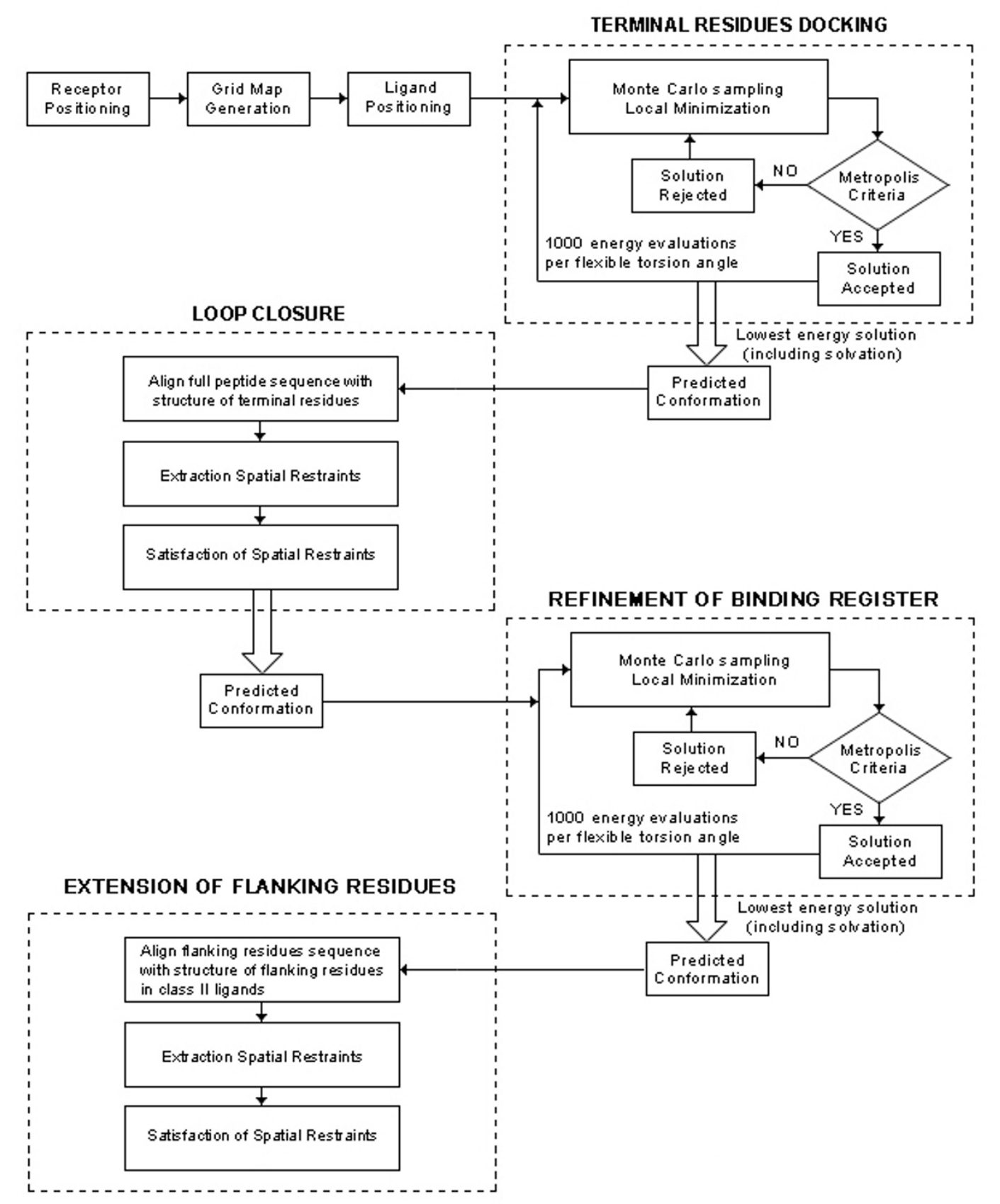

\section{Figure 7}

Flowchart of the four-step docking procedure used in this work. Peptides are docked into DRBI*0402 using a fourstep procedure consisting of: (i) pseudo-Brownian rigid body docking of peptide fragments to the ends of the binding groove, (ii) central loop closure by satisfaction of spatial constraints, (iii) refinement of the backbone and side-chain atoms of the core recognition residues and receptor contact regions and (iv) extension of flanking peptide residues by satisfaction of spatial constraints. 


\section{Accessible Surface Areas}

Solvent accessible surface areas were calculated with the program NACCESS [51] by tracing out the maximum permitted van der Waals' contact that is covered by the center of a water molecule ( $1.40 \AA$ probe radius) as it rolls over the surface of the protein.

\section{Authors' contributions}

JCT carried out the computational simulation studies. JCT and AAS participated in the experimental design, data interpretation and drafted the manuscript.

\section{Acknowledgements}

This work was supported by the Science and Engineering Research Council (SERC) of A*STAR.

\section{References}

I. Beutner EH, Jordon RE: Demonstration of skin antibodies in sera of pemphigus vulgaris patients by indirect immunofluorescent staining. Proc Soc Exp Biol Med 1964, I I7:505-5 I0.

2. Ishii K, Amagai M, Hall RP, Hashimoto T, Takayanagi A, Gamou S, Shimizu N, Nishikawa T: Characterization of autoantibodies in pemphigus using antigen-specific enzyme-linked immunosorbent assays with baculovirus-expressed recombinant desmogleins. J Immunol 1997, I 59:2010-2017.

3. Harman KE, Gratian MJ, Shirlaw PJ, Bhogal BS, Challacombe SJ, Black $M M$ : The transition of pemphigus vulgaris into pemphigus foliaceus: a reflection of changing desmoglein I and 3 autoantibody levels in pemphigus vulgaris. Br J Dermatol 2002, | 46:684-687.

4. Miyagawa S, Amagai M, lida T, Yamamoto Y, Nishikawa T, Shirai T: Late development of antidesmoglein $I$ antibodies in pemphigus vulgaris: correlation with disease progression. BrJ Dermatol 1999, I 4 I: 1084-1087.

5. Tchernev G, Orfanos CE: Antigen mimicry, epitope spreading and the pathogenesis of pemphigus. Tissue Antigens 2006, 68:280-286.

6. Salato VK, Hacker-Foegen MK, Lazarova Z, Fairley JA, Lin MS: Role of intracellular epitope spreading in pemphigus vulgaris. Clin Immunol 2005, I 1 6:54-64.

7. Kalish RS: Pemphigus vulgaris: the other half of the story. J Clin Invest 2000, 106:1433-1435.

8. Chan LS: Epitope spreading in paraneoplastic pemphigus. Autoimmune induction in antibody-mediated blistering skin diseases. Arch Dermatol 2000, 136:663-664.

9. Vanderlugt CJ, Miller SD: Epitope spreading. Curr Opin Immunol 1996, 8:831-836.

10. Chan LS, Vanderlugt CJ, Hashimoto T, Nishikawa T, Zone JJ, Black MM, Wojnarowska F, Stevens SR, Chen M, Fairley JA, Woodley DT, Miller SD, Gordon KB: Epitope spreading: lessons from autoimmune skin diseases. J Invest Dermatol 1998, I 10:103-109.

11. Lin M-S, Swartz SJ, Lopez A, Ding X, Fernandez-Vina MA, Stastny P, Fairley JA, Diaz LA: Development and characterization of desmoglein-3 specific $T$ cells from patients with pemphigus vulgaris. J Clin Invest 1997, 99:3 I-40.

12. Hertl M, Amagai M, Sundaram H, Stanley J, Ishii K, Katz SI: Recognition of desmoglein 3 by autoreactive $T$ cells in pemphigus vulgaris patients and normals. J Invest Dermatol I 998, I I 0:62-66.

13. Wucherpfennig KW, Yu B, Bhol K, Monos DS, Argyris E, Karr RW, Ahmed AR, Strominger JL: Structural basis for major histocompatibility complex (MHC)-linked susceptibility to autoimmunity: charged residues of a single MHC binding pocket confer selective presentation of self-peptides in pemphigus vulgaris. Proc Natl Acad Sci USA 1995, 92: I I935-I 1939.

14. Rizzo C, Fotino M, Zhang Y, Chow S, Spizuoco A, Sinha AA: Direct characterization of human $T$ cells in pemphigus vulgaris reveals elevated autoantigen-specific Th2 activity in association with active disease. Clin Exp Dermatol 2005, 30:535-540.

15. Veldman C, Stauber A, Wassmuth R, Uter W, Schuler G, Hertl M: Dichotomy of autoreactive $\mathrm{Th} \mathrm{I}$ and $\mathrm{Th} 2$ cell responses to desmoglein 3 in patients with pemphigus vulgaris (PV) and healthy carriers of PV-associated HLA class II alleles. J Immunol 2003, 170:635-642.

16. Veldman CM, Gebhard KL, Uter W, Wassmuth R, Grotzinger J, Schultz E, Hertl M: T cell recognition of desmoglein 3 peptides in patients with pemphigus vulgaris and healthy individuals. J Immunol 2004, 172:3883-3892.

17. Veldman C, Pahl A, Beissert S, Hansen W, Buer J, Dieckmann D, Schuler G, Hertl M: Inhibition of the transcription factor Foxp3 converts desmoglein 3-specific type I regulatory $T$ cells into Th2-like cells. J Immunol 2006, 176:3215-3222.

18. Hertl M, Eming R, Veldman C: T cell control in autoimmune bullous skin disorders. J Clin Invest 2006, I 16:1 159-1 I66.

19. Chow S, Rizzo C, Ravitskiy L, Sinha AA: The role of T cells in cutaneous autoimmune disease. Autoimmunity 2005, 38:303-317.

20. Lin MS, Swartz SJ, Lopez A, Ding X, Fairley JA, Diaz LA: T lymphocytes from a subset of patients with pemphigus vulgaris respond to both desmoglein-3 and desmoglein-I. J Invest Dermatol 1997, 109:734-737.

21. Loiseau P, Lecleach L, Prost C, Lepage V, Busson M, Bastuji-Garin S Roujeau JC, Charron D: HLA class II polymorphism contributes to specify desmoglein derived peptides in pemphigus vulgaris and pemphigus foliaceus. J Autoimmun 2000, 15:67-73.

22. Miyagawa S, Higashimine I, lida T, Yamashina Y, Fukumoto T, Shirai T: HLA-DRB I*04 and DRB I*I4 alleles are associated with susceptibility to pemphigus among Japanese. J Invest Dermatol 1997, 109:6/5-618

23. Niizeki $H$, Inoko $H$, Narimatsu $H$, Takata $H$, Sonoda $A$, Tadakuma $T$, Ando A, Tsuji K, Hashimoto T, Nishikawa T: HLA class II antigens are associated with Japanese pemphigus patients. Hum Immunol I99I, 31:246-50.

24. Ahmed AR, Yunis EJ, Khatri K, Wagner R, Notani G, Awdeh Z, Alper CA: Major histocompatibility complex haplotype studies in Ashkenazi Jewish patients with pemphigus vulgaris. Proc Natl Acad Sci USA 1990, 87:7658-7662.

25. Sinha AA, Lopez MT, McDevitt HO: Autoimmune diseases: the failure of self tolerance. Science 1990, 248: | $380-\mid 388$.

26. Sinha AA, Brautbar C, Szafer F, Friedmann A, Tzfoni E, Todd JA, Steinman L, McDevitt HO: A newly characterized HLA DQ beta allele associated with pemphigus vulgaris. Science 1988, 239: 1026-1029.

27. Ahmed AR, Yunis EJ, Khatri K, Wagner R, Notani G, Awdeh Z, Alper $C A$ : Major histocompatibility complex haplotype studies in Ashkenazi Jewish patients with pemphigus vulgaris. Proc Nat| Acad Sci USA 1990, 87:7658-7662.

28. Loiseau P, Lecleach L, Prost C, Lepage V, Busson M, Bastuji-Garin S, Roujeau JC, Charron D: HLA class II polymorphism contributes to specify desmoglein derived peptides in pemphigus vulgaris and pemphigus foliaceus. J Autoimmun 2000, 15:67-73.

29. Lombardi ML, Mercuro O, Ruocco V, Lo Schiavo A, Lombari V, Guerrera V, Pirozzi G, Manzo C: Common human leukocyte antigen alleles in Pemphigus Vulgaris and Pemphigus Foliaceus Italian Patients. J Invest Dermatol 1999, I I 3:107-1 I0.

30. Gonzalez-Escribano MF, Jimenez G, Walter K, Montes M, Perez-Bernal AM, Rodriguez MR, Conejo-Mir JS, Nunez-Roldan A: Distribution of HLA class II alleles among Spanish patients with pemphigus vulgaris. Tissue Antigens 1998, 52:275-278.

31. Glorio R, Rodriguez Costa G, Haas R, Gruber M, Fainboim L, Woscoff A: HLA Haplotypes and Class II Molecular Alleles in Argentinian Patients with Pemphigus Vulgaris. J Cutan Med Surg 2002, 6:422-426.

32. Mobini N, Yunis EJ, Alper CA, Yunis J], Delgado JC, Yunis DE, Firooz A, Dowlati Y, Bahar K, Gregersen PK, Ahmed AR: Identical MHC markers in non-Jewish Iranian and Ashkenazi Jewish patients with pemphigus vulgaris: possible common central Asian ancestral origin. Hum Immunol I997, 57:62-67.

33. Tong JC, Bramson J, Kanduc D, Sinha AA, Ranganathan S: Prediction of desmoglein-3 peptides reveals multiple shared T-cell epitopes in HLA DR4- and DR6-associated pemphigus vulgaris. BMC Bioinformatics 2006, 7:S7.

34. Tong JC, Bramson J, Kanduc D, Chow S, Sinha AA, Ranganathan S: Modeling the bound conformation of pemphigus vulgarisassociated peptides to MHC class II DR and DQ alleles. Immunome Res 2006, 2:1. 
35. Tong JC, Tan TW, Ranganathan S: Modeling the structure of bound peptide ligands to major histocompatibility complex. Protein Sci 2004, I 3:2523-2532.

36. Tong JC, Zhang ZH, August JT, Brusic V, Tan TW, Ranganathan S: In silico characterization of immunogenic epitopes presented by HLA-Cw*040 I. Immunome Res in press.

37. Tong JC, Zhang GL, Tan TW, August JT, Brusic V, Ranganathan S: Prediction of HLA-DQ3.2 $\beta$ ligands: Evidence of multiple registers in class II binding peptides. Bioinformatics 2006, 22: $1232-1238$.

38. Lucchese A, Mittelman A, Tessitore L, Serpico R, Sinha AA, Kanduc $D$ : Proteomic definition of a desmoglein linear determinant common to Pemphigus vulgaris and Pemphigus foliaceous. J Transl Med 2006, 4:37.

39. Lin MS, Swartz SJ, Lopez A, Ding X, Fairley JA, Diaz LA: T lymphocytes from a subset of patients with pemphigus vulgaris respond to both desmoglein-3 and desmoglein-I. J Invest Dermatol 1997, 109:734-737.

40. Seishima M, Esaki C, Osada K, Mori S, Hashimoto T, Kitajima Y: Pemphigus IgG, but not bullous pemphigoid IgG, causes a transient increase in intracellular calcium and inositol $1,4,5$ triphosphate in DJM-I cells, a squamous cell carcinoma line. J Invest Dermatol 1995, 104:33-37.

41. Robinson J, Waller MJ, Parham P, de Groot N, Bontrop R, Kennedy LJ, Stoehr P, Marsh SG: IMGT/HLA and IMGT/MHC: sequence databases for the study of the major histocompatibility complex. Nucleic Acids Res 2003, 3 I:3 I I-3 |4.

42. Boeckmann B, Bairoch A, Apweiler R, Blatter MC, Estreicher A, Gasteiger E, Martin MJ, Michoud K, O'Donovan C, Phan I, Pilbout S, Schneider M: The SWISS-PROT protein knowledgebase and its supplement TrEMBL in 2003. Nucleic Acids Res 2003, 3 I:365-370.

43. Berman HM, Westbrook J, Feng Z, Gilliland G, Bhat TN, Weissiq H, Shindyalov IN, Bourne PE: The Protein Data Bank. Nucleic Acids Res 2000, 28:235-242

44. Altschul SF, Gish W, Miller W, Myers EW, Lipman DJ: Basic local alignment search tool. I Mol Biol 1990, 21 5:403-4I0.

45. Sali A, Blundell TL: Comparative protein modelling by satisfaction of spatial restraints. I Mol Biol 1993, 234:779-8I5.

46. MacKerell AD, Bashford D, Bellott M, Dunbrack RL, Evanseck JD, Field MJ, Fischer S, Gao J, Guo H, Ha S, Joseph-McCarthy D, Kuchnir L, Kuczera K, Lau FTK, Mattos C, Michnick S, Ngo T, Nguyen DT, Prodhom B, Reiher WE, Roux B, Schlenkrich M, Smith JC, Stote R, Straub J, Watanabe M, Wiórkiewicz-Kuczera J, Yin D, Karplus M: Allatom empirical potential for molecular modeling and dynamics studies of proteins. I Phys Chem 1998, B I 02:3586-36I7.

47. Abagyan R, Totrov M, Kuznetsov D: ICM - a new method for protein modeling and design: Applications to docking and structure prediction from the distorted native conformation. Comput Chem 1994, I 5:488-506.

48. Abagyan RA, Totrov M: Ab initio folding of peptides by the optimal-bias Monte Carlo minimization procedure. J Comput Phys |999, I 5 |:402-42 |.

49. Srinivasan KN, Zhang GL, Khan AM, August JT, Brusic V: Prediction of class I T-cell epitopes: evidence of presence of immunological hot spots inside antigens. Bioinformatics 2004, 20:i297-i302.

50. Zhang GL, Khan AM, Srinivasan KN, August JT, Brusic V: MULTIPRED: a computational system for prediction of promiscuous HLA binding peptides. Nucleic Acids Res 2005 33:WI72-WI79.

5I. Hubbard SJ, Thornton JM: NACCESS. Univ Coll, London; 1993.
Publish with Bio Med Central and every scientist can read your work free of charge

"BioMed Central will be the most significant development for disseminating the results of biomedical research in our lifetime. "

Sir Paul Nurse, Cancer Research UK

Your research papers will be:

- available free of charge to the entire biomedical community

- peer reviewed and published immediately upon acceptance

- cited in PubMed and archived on PubMed Central

- yours - you keep the copyright

Submit your manuscript here:

http://www.biomedcentral.com/info/publishing_adv.asp
BioMedcentral 